Type of the paper: Research articles

Running title: Production of anticarcinogenic surfactin by Bacillus pumilus HY1

\title{
Anticancer effect of the five surfactin isomers produced during cheonggukjang fermentation by Bacillus pumilus HY1 isolated from Korean traditional fermented soy sauce
}

\section{Su Young Hong ${ }^{1}$, Dong Hee Lee ${ }^{2}$, Jin Hwan Lee ${ }^{3}$, Kye Man Cho ${ }^{4 *}$}

1 Food Science Research Institute, SBT Business Division, Kolmar BNH Inc., Sejong 30003, Republic of Korea

2 Industry Academy Cooperation Foundation, Andong National University, Andong, 36729, Republic of Korea

3 Department of Life Resources Industry, Dong-A University, Busan 49315, Republic of Korea

4 Department of Food Science, Gyeongnam National University of Science and Technology, Jinju 52725, Republic of Korea

* Correspondence: kmcho@gntech.ac.kr (K.M. C.).

Tel.: +82-55-751-3272 (K.M.C.) 
Abstract: The cyclic lipopeptide produced from Bacillus pumilus strain HY1 was isolated from Korean soybean sauce. The chemical structures of the surfactin isomers were analyzed using matrix-assisted laser desorption ionization time-of-flight mass spectrometry (MALDITOF MS) and electrospray ionization tandem mass spectrometry (ESI-MS/MS). The five potential surfactin isoforms were detected with protonated masses of $m / z$ 994.7, 1,008.7, 1022.7, 1036.7, and 1,050.7 and different structures in combination with $\mathrm{Na}^{+}, \mathrm{K}^{+}$, and $\mathrm{Ca}^{2+}$ ions. ESI-MS/MS analysis revealed that the isolated surfactin possessed the precise amino acid sequence LLVDLL and hydroxyl fatty acids with 12 to 16 carbons. The surfactin content during cheonggukjang fermentation increased from 0.3 to $51.2 \mathrm{mg} / \mathrm{kg}$ over $60 \mathrm{~h}$ of fermentation. The surfactin extraction of cheonggukjang inhibited the growth of two cancer cell lines. The growth of both MCF-7 and Caco-2 cells was strongly inhibited with 100 $\mu \mathrm{g} / \mu \mathrm{L}$ surfactin. These results suggest that surfactins produced from strain HY1 have anticancer properties.

Keywords: Bacillus pumilus HY1; Solid-state fermentation; Cheonggukjang; Surfactin; Mass spectrum; Anticancer effect

Abbreviations: CLP, cyclic lipopeptide; SEC, surfactin extraction of cheonggukjang; TS, tryptic soy; No. 3, number 3; MTT, 3-(4,5-dimethylthiazol-2-yl)-2,5-diphenyltetrazolium 
bromide; EDTA, ethylenediaminetetraacetic acid; DMSO, dimethyl sulfoxide; FBS, fetal bovine serum; MALDI-TOF MS, matrix-assisted laser desorption ionization time-of-flight mass spectrometry; ESI-MS/MS, electrospray ionization tandem mass spectrometry; HPLC, high performance liquid chromatography 


\section{Introduction}

Surfactins are cyclic lipopeptide (CLP) biosurfactants produced by several Bacillus strains including B. subtilis, B. amyloliquefaciens, B. pumilus, B. licheniformis and B. mojavensis [1-5]. Surfactin is produced non-ribosomally by multienzyme complexes called nonribosomal peptide synthetases [6]. Small microbial peptides produced via non-ribosomal pathways are of great interest, reflecting their many potent biological activities and growing economic value such as drugs or food additives [7]. Surfactin is composed of one $\beta$-hydroxy fatty acid molecule with a long fatty acid moiety, linked to a seven amino acid peptide to form a lactone ring [8]. Surfactin exhibits diverse biological activities, including antitumoral [4,9,10], antiviral [11], antibacterial [12], antimycoplasmal [13], hemolytic [14], and fibrinolytic activities [15]. In addition, surfactin inhibits fibrin clot formation [16], phosphodiesterase activity [17], cyclic adenosine monophosphate (cAMP) signaling [17], and platelet and spleen cytosolic phospholipase A2 (PLA2) activity [18].

There are several types of traditional Korean fermented soybean foods including meju (soybean cake), doenjang (soybean paste), kanjang (soybean sauce), and cheonggukjang (soybean cook). Fermented soybean is a good source of protein, hydrolyzed peptides, and lipids, and many Koreans consume soybeans for their health benefits, which include reducing arterial stiffness. Several reports have shown the antioxidant, antimicrobial, 
antigenotoxic, blood pressure lowering and antidiabetic activities of cheonggukjang [19,20]. In a previous study, we reported the production of surfactin from a potential probiotic, Bacillus subtilis CSY191, and the purified surfactin exhibited anticancer activity against human breast cancer MCF-7 cells [4]. The identification and separation of the various beneficial components in chenoggukjang enables a demonstration of their health benefits and promotes the development of distinct functional products for the food industry.

In the present study, surfactin was purified from strain B. pumilus HY1, a bacterial strain commonly detected in traditional Korean fermented soy sauce (kanjang). The surfactin was fractionated through TLC and RP-HPLC and characterized using mass spectrometry. The concentration of surfactin during the fermentation of cheonggukjang by B. pumilus HY1 was determined. Finally, the antiproliferative activity of surfactin extraction of cheonggukjang against the cancer cell lines MCF-7 (human breast cancer line) and Caco-2 (human intestinal cancer line) was studied.

\section{Materials and methods}

2.1. Microorganism, soybean, media, cells, and regents

The CLP-producing B. pumilus strain HY1 was isolated from Korean soybean sauce (kanjang) collected from Daegok in Jinju, Korea [21]. Soybean cultivar, namely Taekwang, 
was harvested and provided in 2013 from the National Institute of Crop Science of the Rural Development Administration in Korea. The tryptic soy (TS), number 3 (No. 3), and DMEM/F-12 media were purchased from Becton Dickinson Co. (Difco, Sparks, MD, USA). MCF-7 (human breast cancer cell line) and Caco-2 (human intestinal cancer cell line) were obtained from the Korean Cell Line Bank in Seoul, Korea. Sheep blood, surfactin standards (consisting of $\mathrm{C}_{13}$ and $\mathrm{C}_{15} \beta$-amino acids), glycerol, triglycerol, $\alpha$-cyano-4-hydroxycinnamic acid, trifluoroacetic acid, triethylamine, phenylisothiocyanate, 3-(4,5-dimethylthiazol-2-yl)2,5-diphenyltetrazolium bromide (MTT), ethylenediaminetetraacetic acid (EDTA), dimethyl sulfoxide (DMSO), fetal bovine serum (FBS), penicillin, and streptomycin were obtained from the Sigma-Aldrich, Inc. (Merck KGaA, Darmstadt, Germany). The high performance liquid chromatography (HPLC)-grade chloroform, methanol, water and acetonitrile were purchased from Fisher Scientific International, Inc. (Fairlawn, NJ, USA). Other regents and solvents used the analytical grade (Sigma-Aldrich, Inc.).

\subsection{Isolation and purification of surfactin}

Surfactin was isolated from strain HY1 according to Lee et al. [4]. The cells were grown in No. 3 medium at $30^{\circ} \mathrm{C}$. After cultivation for 3 days, the supernatant was collected through centrifugation and adjusted to $\mathrm{pH} 2.0$ using concentrated $\mathrm{HCl}$. The precipitate was collected through centrifugation and extracted three times with methanol. The Methanolic extracts 
were separated using thin layer chromatography on silica gel 60 plates (Merck KGaA, Darmstadt, Germany). Chloroform/methanol/water $(65: 25: 5, \mathrm{v} / \mathrm{v} / \mathrm{v})$ was used as the developing solvent. The various spots were visualized by charring after spraying with concentrated sulfuric acid. To isolate the surfactin fraction, the corresponding spots were scratched from the thin-layer chromatography (TLC) plate and the silica gel material was extracted using methanol. For further purification, $1 \mathrm{mg}$ of extract was subjected to HPLC (LC-908, JAIGEL-1H column, Japan Analytical Industry, Japan) and eluted with acetonitrile/water $(1: 1, \mathrm{v} / \mathrm{v})$ as the mobile phase at a flow rate of $2.5 \mathrm{~mL} / \mathrm{min}$.

\subsection{Mass spectrometric analysis of surfactin isoforms}

The purified CLP was analyzed using matrix-assisted laser desorption ionization timeof-flight mass spectrometry (MALDI-TOF MS, Vg-Instruments, Manchester, UK) and inductively coupled plasma mass spectrometer (ICP MS; Elan Drc II, PerkinElmer Inc., New York, NY, USA). Five micrograms of sample were dissolved in DMSO-glycerol and introduced on a copper probe tip using a mixture of glycerol and triglycerol as a matrix. A saturated solution of $\alpha$-cyano-4-hydroxycinnamic acid in $70 \%$ acetonitrile/0.1\% trifluoroacetic acid (1:1, v/v) was mixed with an equal volume of sample for the MALDITOF mass analysis. One microliter of the sample (2 to $3 \rho \mathrm{mol}$ ) was deposited onto a sample plate and air-dried. Ions were accelerated with a voltage of $20 \mathrm{kV}$. The positive-ion and 
reflector mode was applied.

\subsection{Amino acid and mineral analysis}

The amino acid and mineral composition analysis of the purified CLP wines were determined according to methods previously described by Cho et al. [22]. The amino acid of the purified CLP was sequenced using electrospray ionization tandem mass spectrometry (ESI-MS/MS; Finnigan-MAT TSQ 700, San Jose, USA). The sample was dissolved in 50\% aqueous methanol containing $1 \%$ formic acid prior to injection into the mass spectrometer. ESI-MS/MS spectra were acquired after inducing collisions between precursor ions and nitrogen collision gas at acceleration voltages of $50 \mathrm{~V}$.

\subsection{Preparation and fermentation of cheonggukjang}

Soybean $(1 \mathrm{~kg})$ was washed and soaked with three times volume of tap water at $20 \pm 2{ }^{\circ} \mathrm{C}$ for $12 \mathrm{~h}$, followed by steaming for $30 \mathrm{~min}$ at $121 \pm 1^{\circ} \mathrm{C}$. The steamed soybeans were incubated at $37^{\circ} \mathrm{C}$ for $1 \mathrm{~h}$ to cool down the sample. The cooked soybeans were subsequently inoculated with 5\% (v/w) HY1 (7.43 log CFU/mL), fermented for $60 \mathrm{~h}$ at $37 \pm 2{ }^{\circ} \mathrm{C}$ and sampled at $0,12,24,36,48$, and $60 \mathrm{~h}$. The growth of strain HY1 during fermentation was determined as viable cell counts at each time point. One gram of sample was mixed with 9 $\mathrm{ml}$ of $0.85 \% \mathrm{NaCl}$ solution, and the dilutions were spread onto TS agar plates. Colonies were 
counted after incubation at $37{ }^{\circ} \mathrm{C}$ for $24 \mathrm{~h}$ [19].

\subsection{Extraction and analysis of surfactin}

The extraction and analysis of surfactin were performed according to Lee et al. [4] methods. Ten grams of ground cheonggukjang was extracted using $30 \mathrm{~mL}$ of methanol and adjusted to $\mathrm{pH} 2.0$ with concentrated $\mathrm{HCl}$ by shaking $(160 \mathrm{rpm})$ at $30{ }^{\circ} \mathrm{C}$ for $12 \mathrm{~h}$. The extract was filtered through Whatman No. 2 filter paper and dried under a vacuum. The dried material was redissolved in $10 \mathrm{~mL}$ of $80 \%$ methanol and filtered through a $0.45-\mu \mathrm{m}$ Millipore PVDF filter (Schleicher \& Schuell, GmbH, Dassel, Germany). The filtrate was used for HPLC analysis to determine the surfactin concentration during the fermentation of cheonggukjang. The injection volume of the sample was $20 \mu \mathrm{L}$. The surfactin was analyzed through HPLC (Perkin-Elmer 200 series, Perkin-Elmer Corp., Norwalk, CT, USA) using an RP C18 column $(4.6 \times 250 \mathrm{~mm}, 5 \mu \mathrm{m}$, Waters Corp., Milford, MA, USA). The acetonitrile:water $(1: 1, \mathrm{v} / \mathrm{v})$ was eluted at a flow rate of $1 \mathrm{Ml} / \mathrm{min}$ at $40^{\circ} \mathrm{C}$. Surfactin was measured at $210 \mathrm{~nm}$ using a UV detector (Perkin-Elmer UV 200 series, Perkin-Elmer Corp., Norwalk, CT, USA). The concentration of surfactin was determined using a standard curve with standard solutions at $25,50,75$, and $100 \mu \mathrm{g} / \mathrm{mL}$.

\subsection{MTT assay}


MCF-7 and Caco-2 cells were used as cancer cell lines for the MTT assay. The cells were cultured in DMEM/F-12 medium supplemented with 10\% FBS and 1\% penicillinstreptomycin. The MTT assay was determined by the method of Lee et al. [4]. Briefly, The two cancer cells were dissociated with $0.05 \%$ trypsin- $0.02 \%$ EDTA, and $180 \mu \mathrm{L}$ of the cell suspension $\left(1 \times 10^{4}\right.$ cells $\left./ \mathrm{mL}\right)$ was seeded onto 48 -well microtiter plates and treated with various concentrations of surfactin extraction of cheonggukjang (SEC) (20 $\mu \mathrm{L})$. After incubation for $24 \mathrm{~h}, 20 \mu \mathrm{L}$ of MTT solution $(2.5 \mathrm{mg} / \mathrm{mL}$ PBS $)$ was added. The formazan dye was solubilized by adding $150 \mu \mathrm{L}$ of DMSO to each well, followed by gentle shaking. The optical densities were read on an ELISA reader (680; Bio-Rad, Tokyo, Japan) at $540 \mathrm{~nm}$.

\section{Results}

\subsection{Isolation of surfactin from B. pumilus $H Y 1$}

The surfactin was produced during the fermentation of high producer B. pumilus HY1 in No. 3 medium. Acid-precipitated and methanol-extracted bacterial cells were concentrated and chromatographed. The TLC of the obtained CLP material was performed on silica gel 60 using solvent (chloroform/methanol/water $=65: 25: 4, \mathrm{v} / \mathrm{v} / \mathrm{v}$ ) as the mobile phase, which showed a broad spot with an $R f$ value of 0.5 to 0.55 . Analytical gel filtration of the entire surfactin mixture was performed using surfactin from B. subtilis (Sigma-Aldrich, Inc.) as a 
size marker, yielding a molecular mass of 1,036. Reverse phase HPLC was used to analyze surfactin, followed by purification at $214 \mathrm{~nm}$ (Fig. 1).

\subsection{Mass spectrum of surfactin by B. pumilus HY1}

The purified surfactin was analyzed and identified using MALDI-TOF MS, ICP MS, and ESI MS/MS. The MALDI-TOF MS displayed $[\mathrm{M}+\mathrm{H}]^{+}$peaks at $\mathrm{m} / z$, 994.7, 1,008.7, $1022.7,1036.7$, and $1,050.7$, and these peaks were separated by $m / z 14$. The MALDI-TOF MS displayed the groups of peaks at $m / z$ 1016.58, 1030.58, 1044.61, 1058.62, and 1072.62, which could reflect the isoform ensembles of surfactin (Fig 2). The surfactin contained a mixture of structural analogs with a mass differences of 14 Da. Most of the peaks could reflect essentially pure surfactin isoforms using MALDI-TOF MS to detect the distribution of the molecular ions in these fractions and the assignment of the ions to different surfactin species. Based on the mass spectrometric data, the surfactin isoforms were eluted according to their hydrophobicities. For the large peak appearing in fractions in front of the five main peaks, the main parent ions detected at $m / z$ 1016.5811, 1030.5833, 1044.6104, 1058.6250, and 1072.6289 were attributed to a small amount of the sodium adduct of a valine-7 surfactin. The low mass peaks at $m / z 1032.5869,1046.5831,1060.6001,1074.6023$, and 1088.6222 represented the potassium adduct of these species. The other mass peaks at $m / z$ 1033.5630, 1047.5841, 1061.6024, 1075.5997, and 1089.8287 represented the calcium adduct of these 
species. These peaks contained essentially pure $C_{12}$ to $C_{16}$ surfactin species, respectively. The purified surfactin was analyzed using ICP MS, and potassium and calcium ions were detected (Table 1). These results were consistent with those of the MALDI-TOF MS analysis, suggesting the presence of ions. The four amino acids Asp, Glu, Val, and Leu at a ratio 1:1:1:4 were detected in the purified HPLC fraction of surfactin after hydrolysis and derivatization using Marfey's reagent. The amino acid composition corresponds to that of surfactin. For the detailed analysis, the peptide sequence of surfactin was deduced after interpreting the ESI-MS/MS spectrum of the precursor ions $\mathrm{m} / \mathrm{z} 994.7081,1,008.7334$, $1,022.7391,1,036.7528$, and $1,050.7487$ assuming the preferential cleavage of the ring opening in the lactone bond in the collision chamber (Fig. 3).

3.3. Changes in viable cell number of B. pumilus HY1 and surfactin concentration during cheonggukjang fermentation.

Changes in the bacterial viable cell numbers and surfactin concentration during the fermentation of cheonggukjang are shown in Fig 4. The level of viable cells in fermented cheonggukjang range from $3.0 \log \mathrm{CFU} / \mathrm{mL}(0 \mathrm{~h})$ to $11.7 \log \mathrm{CFU} / \mathrm{mL}(60 \mathrm{~h})$. Correspondingly, the concentration of surfactin in cheonggukjang fermentation increased from $0.3 \mathrm{mg} / \mathrm{kg}$ in the initial stage to $51.2 \mathrm{mg} / \mathrm{kg}$ after $60 \mathrm{~h}$. The surfactin concentration peaked at $48.4 \mathrm{mg} / \mathrm{kg}$ at $48 \mathrm{~h}$ and increased slightly at the end of fermentation. 


\subsection{Effect of SEC on the growth of cancer cells}

We used the well-characterized MTT assay to assess the metabolic activity of cells, to determine whether surfactin could inhibit the growth of MCF-7 and Caco-2 cells, two cancer cell lines. MCF-7 and Caco-2 cells were incubated with SEC at concentrations ranging from 0 to $120 \mu \mathrm{g} / \mu \mathrm{L}$ of surfactin, as shown in Fig. 5. The SEC inhibited the growth of cells in a concentration-dependent manner. Morphological changes provide the most direct criteria for recognizing the apoptotic process. As shown in Fig. 6, apoptotic cells were observed at $24 \mathrm{~h}$ after exposure to SEC (100 $\mu \mathrm{g} / \mu \mathrm{L}$ of surfactin).

\section{Discussion}

In the present study, we investigated the purification of surfactin from B. pumilus HY1, the CLP (such as iturin and surfactin) producing strain isolated from kanjang [21]. The surfactins were fractionated using high resolution RP-HPLC and determined by MADI-TOF MS and ESI-MS/MS. Also, the surfactin concentration was confirmed during cheonggukjang fermentation by B. pumilus HY1, and the anticancer effect of SECs against MCF-7 and Caco-2 cells was examined. Surfactin is a cyclic heptalipopeptide with the sequence GluLeu-Leu-Val-Asp-Leu, a terminal Leu linked to a $\beta$-hydroxy fatty acid residue, with amide 
(Glu) and lactone (Leu) bonds forming a cyclic structure. Three isoforms in natural surfactin with substitution of the L-Leu in position 7 of the main product by L-Val and L-Ile were characterized using 2D NMR spectroscopy in combination with chemical analysis [23]. However, the surfactin of B. pumilus HY1 possessed five potential isoforms with protonated masses of $994.7,1,008.7,1022.7,1036.7$, and $1,050.7 \mathrm{~m} / \mathrm{z}$ and different structures in combination with $\mathrm{Na}^{+}, \mathrm{K}^{+}$, and $\mathrm{Ca}^{2+}$ ions (Fig. 2). These results are consistent with data obtained from the analysis of the amino acid composition and showed that surfactin purified from $B$. pumilus $\mathrm{HY} 1$ was identical to surfactin produced from $B$. subtilis and $B$. amyloliquefaciens. ESI-MS/MS analysis revealed that the structure of fatty acid was simulated, and the results indicated that surfactin was determined as a homologous series of hydroxy fatty acids with $12,13,14,15$, and 16 carbon atoms (Table 1). The biological activity of surfactins from Bacillus sp. depends both on the peptide ring and the nature of their lipid moieties. The hemolytic activity of surfactin was enhanced by the increase in the number of carbon atoms in the fatty acid side chains, and this enhanced activity likely reflects stronger interactions with biomembranes [24].

In previous, the biological activities of surfactin occur via interactions with cellular membranes and it may have detergent-like effect on cell membranes [4,10]. The many researches have penetrated that hydrophobic residues of the peptide moiety permeate into the membrane interface, leading to membrane permeabilization but not surface effect $[4,24]$. At 
higher concentrations, this detergent-like activity could stabilize leaks due to the compound's ability to generate multiple-structured polymers [24]. Although it is not yet clear whether a sub-optimal concentration ( $\mathrm{IC}_{50} 100 \mu \mathrm{g} / \mu \mathrm{L}$ ) of surfactin affects the cell morphology (Fig. 6), surfactin-treated cells appeared to be leaky and/or lysed. Meta et al. [25]. proposed that surfactin may disturb a biochemical reaction(s) that occurs at a specific membrane site perhaps via the long chain fatty acid, acting as a pseudosubstrate (similar to PIP3 or a farnesyl group) for either the PI3K/Akt or farnesyltransferase/Ras/ERK pathways, which promote apoptosis. In a previous study, Cao et al. [26] suggested that surfactin induces apoptosis in human breast cancer MCF-7 cells through a ROS/JNK-mediated mitochondrial/caspase pathway and the surfactin has notable anti-tumor effects on MCF-7 cells; however, there was no obvious cytotoxicity on normal cells.

Carbohydrate and lipoprotein extracts from edible mushrooms and several bacterial cells were reported for their growth inhibition towards malignant cancer cells [27]. A number of recent studies have focused on the anticancer activities of CLP. Kameda et al. [9] provided the first evidence that CLP has anticancer activity based on the fact that extracted CLP from B. natto KMD 2311 can induce apoptosis of cancer cells. Zhao et al. [28] reported that exposure of B16 cells to lipoproteins resulted in chromatin condensation, DNA fragmentation and sub-G1 arrest. Sudo et al. [29] examined lipoproteins for their ability to inhibit growth and induce differentiation of HL60 human promyelocytic leukemia cells, and 
Wakamatsu et al. [27] discovered that lipoproteins induce neuronal differentiation in PC12 cells and provided the groundwork for the use of microbial extracellular CLP as a novel reagent for the treatment of cancer cells. Wang et al. [30] reported that a new CLP purified from B. subtilis subsp. natto T-2 dose-dependently inhibited the growth of human leukemia K562 cells. Kim et al. [10] examined the effects of surfactin on the proliferation of LoVo cells (a human colon carcinoma cell line) and suggested that surfactin may have anticancer properties as a result of its ability to down-regulate the cell cycle and suppress cell survival. In addition, Cao et al. [31] reported that the purified surfactin from Bacillus natto TK-1 induced the time-dependent apoptosis of human breast cancer MCF-7 cells through cell cycle arrest at the G2/M phase. These results suggest that the surfactin of B. pumilus HY1 has potential value as a novel anti-tumor agent.

The content of surfactin increased from $0.3 \mathrm{mg} / \mathrm{kg}$ on day 0 to a final content of 51.2 $\mathrm{mg} / \mathrm{kg}$, corresponding to the cell concentration increases of 3.0-11.7 log CFU/g during cheonggukjang fermentation (Fig. 4). Several authors have studied the production of surfactin from solid-state fermentation $[4,10,32,33]$. This process is associated with the production of a higher concentration of surfactin in solid-state fermentation, which occurs in the manufacturing of soybean-fermented food [10]. In particular, Slvinski et al. [34] studied the production of surfactin through B. pumilus UFPEDA 448 in solid-state fermentation, using a medium based on okara with the addition of sugarcane bagasse as a bulking agent. 
This result shows that the concentration of surfactin is proportional to the concentration of cells because surfactin is produced from Bacillus sp.

The cheonggukjang is reported to have anticancer, blood pressure reduction, hypocholesterolemic, and fibrinolytic properties [4,19]. However, although these effects of cheonggukjang have been extensively studied, little is known about its potential antitumoral activities. In addition, we previously reported that the surfactin content, during cheonggukjang fermentation with $B$. subtilis CSY191, increased from 0.3 to $48.2 \mathrm{mg} / \mathrm{kg}$ over $60 \mathrm{~h}$ of fermentation, while the level of anticancer activity increased from 2.6- to 5.1-fold [4]. These findings will improve the quality of Korean traditional soybean fermented foods as one of the best functional foods because of their wide variety of antitumor, antimicrobial, antifungal, and antiviral activities.

In conclusions, we identified five potential isoforms of surfactin from B. pumilus HY1. Surfactin is a significant component of the stain HY1-fermented cheonggukjang with surfactin reaching $51.2 \mathrm{mg} / \mathrm{kg}$ at the end of fermentation $(60 \mathrm{~h})$. Surfactin extraction of cheonggukjang had antiproliferative activity against two cancer cells lines. Further studies are needed to define the in vivo anticancer effects of surfactin and clarify its precise molecular mechanism(s) of action.

Author Contributions: S.Y.H. performed the experiments, analyzed the data, and wrote the 
paper. D.H.L. commented the manuscript. K.M.C. conceived and designed the experiments, interpreted the data and prepared the manuscript. All authors have read and approved the manuscript.

Funding: This research was supported by the Basic Science Research Program through the National Research Foundation of Korea (NRF) funded (grant number 2010-0022508) by the Ministry of Education and supported by Agro \& Bio-industry Technology Development Program (Grant No. 314021-03), Ministry of Agriculture, Food and Rural Affairs, Republic of Korea., Republic of Korea.

Conflict of interests: The authors declare that there is no conflict of interests regarding the publication of this paper.

\section{Reference}

1. From, C.; Hormazabal, V.; Hardy, S.P.; Granum, P.E. Cytotoxicity in Bacillus mojavensis is abolished following loss of surfactin synthesis: implications for assessment of toxicity and food poisoning potential. Int. J. Food Microbiol. 2007, 117, 43-49.

2. Kalinovskaya, N.I.; Kuznetsova, T.A.; Ivanova, E.P.; Romanenko, L.A.; Voinov, V.G.; Huth, F.; Laatsch, H. Characterization of surfactin-like cyclic depsipeptides synthesized by Bacillus pumilus from ascidian Halocynthia aurantium. Marine Biotechnol. 2002, 4, 
179-188.

3. Koumoutsi, A.; Chen, X.H.; Henne, A.; Liesegang, H.; Hitzeroth, G.; Franke, P.; Vater, J.; Borriss, R. Structural and functional characterization of gene clusters directing nonribosomal synthesis of bioactive cyclic lipopeptides in Bacillus amyloliquefaciens strain FZB42. J. Bacteriol. 2004, 186, 1084-1096.

4. Lee, J.H.; Nam, S.H.; Seo, W.T.; Yun, H.D.; Hong, S.Y.; Kim, M.K.; Cho, K.M. The production of surfactin during the fermentation of cheonggukjang by potential probiotic Bacillus subtilis CSY191 and the resultant growth suppression of MCF-7 human breast cancer cells. Food Chem. 2012, 131, 1347-1354.

5. Thaniyavarn, J.; Roongsawang, N.; Kameyama, T.; Haruki, M.; Imanaka, T.; Morikawa, M.; Kanaya, S. Production and characterization of biosurfactants from Bacillus licheniformis F2.2. Biosci. Biotechnol. Biochem. 2003, 67, 1239-1244.

6. Schwarzer, D.; Finking, R.; Marahiel, M.A. Nonribosomal peptides: from genes to products. Nat. Prod. Rep. 2003, 20, 275-287.

7. Symmank, H.; Franke, P.; Saenger, W.; Bernhard, F. Modification of biologically active peptides: production of a novel lipohexapeptide after engineering of Bacillus subtilis surfactin synthetase. Protein Eng. 2002, 15, 913-921.

8. Hue, N.; Serani, L.; Laprevote, O. Structural investigation of cyclic peptidolipids from Bacillus subtilis by high-energy tandem mass spectrometry. Rapid Commun. Mass 
Spectrom. 2001, 15, 203-209.

9. Kameda, Y.; Oira, S.; Matsui, K.; Kanatomo, S.; Hase, T. Antitumor activity of Bacillus natto. V. Isolation and characterization of surfactin in the culture medium of Bacillus natto KMD 2311. Chem. Pharm. Bul. 1974, 22, 938-44.

10. Kim, K.M.; Lee, J.Y.; Kim, C.K.; Kang, J.S. Isolation and characterization of surfactin produced by Bacillus polyfermenticus KJS-2. Arch. Pharm. Res. 2009, 32, 711-715.

11. Vollenbroich, D.; Ozel, M.; Vater, J.; Kamp, R. M.; Pauli, G. Mechanism of inactivation of enveloped viruses by the biosurfactant surfactin from Bacillus subtilis. Biologicals 1997, 25, 289-297.

12. Tsukagoshi, N.; Tamura, G.; Arima, K. A novel protoplast bursting factor (surfactin) obtained from Bacillus subtilis IAM1213. I. The effects of surfactin on Bacilllus megaterium KM. Biochim. Biophys. Acta 1970, 196, 204-210.

13. Hwang, M.H.; Chang, Z.Q.; Kang, E.H.; Lim, J.H.; Yun, H,I.; Rhee, M.H.; Jeong, K.S.; Park, S.C. Surfactin C inhibits Mycoplasma hyopneumoniae-induced transcription of proinflammtory cytokines and nitric oxide production in murin RAW 264.7 cells. Biotechnol. Lett. 2008, 30, 229-233.

14. Morán, A.C.; Martínez, M.A.; Siñeriz, F. Quantification of surfactin in culture supernatants by hemolytic activity. Biotechnol. Lett. 2002, 24, 177-180.

15. Kikuchi, T.; Hasumi, K. Enhancement of plasminogen activation by surfactin C: 
augmentation of fibrinolysis in vitro and in vivo. Biochim. Biophys. Acta 2002, 29, 234245.

16. Arima, K.; Kakinuma, A.; Tamura, G. Surfactin, a crystalline peptide lipid surfactant produced by Bacillus subtilis: isolation, characterization and its inhibition of fibrin clot formation. Biochem. Biophys. Res. Commun. 1968, 31, 488-494.

17. Hosono, K.; Suzuki, H. Acylpeptides, the inhibition of cyclic adenosine 3',5' monophosphate phosphodiesterase. III. Inhibition of cyclic AMP phosphodiesterase. $J$. Antibiot. 1983, 36, 679-683.

18. Kim, K.; Jung, S.Y.; Lee, D.K.; Jung, J.; Park, J.K.; Kim, D.K.; Lee, C. Suppression of inflammatory responses by surfactin, a selective inhibitor of platelet cytosolic phospholipase A2. Biochem Pharmacol. 1998, 55, 975-985.

19. Cho, K.M.; Hong, S.Y.; Math, R.K.; Lee, J.H.; Kambiranda, D.M.; Kim, J.M.; Islam, S.M.A.; Yun, M.G.; Cho, J.J.; Lim, W.J.; et al. Biotransformation of phenolics (isoflavones, flavanols and phenolic acids) during the fermentation of cheonggukjang by Bacillus pumilus HY1. Food Chem. 2009, 114, 413-419.

20. Cho, K.M.; Lee, J.H.; Yun, H.D.; Ahn, B.Y.; Kim, H.; Seo, W.T. Changes of phytochemical constituents (isoflavones, flavanols, and phenolic acids) during cheonggukjang soybeans fermentation using potential probiotics Bacillus subtilis CS90. J. Food Compost. Anal. 2011, 24, 402-410. 
21. Cho, K.M.; Math, R.K.; Hong, S.Y.; Islam, S.M.A.; Mandanna D.K.; Cho, J.J.; Yun, M.G.; Kim, J.M.; Yun, H.D. Iturin produced by Bacillus pumilus HY1 from Korean soybean sauce (kanjang) inhibits of aflatoxin producing fungi. Food Cont. 2009, 20, $402-406$.

22. Cho, S.J.; Hong, S.Y.; Kim, J.Y.; Park, S.R.; Kim, M.K.; Lim, W.J.; Shin, E.C.; Kim, E.J.; Cho, Y.U.; Yun, H.D. Endophytic Bacillus sp. CY22 from a balloon flower (Platycodon grandiflorum) produces surfactin isoforms. J. Microbiol. Biotechnol. 2003, $13,859-865$.

23. Baumgart, F.; Kluge, C.; Ullrich, B.; Vater, J.; Ziessow, D. Identification of amino acid substitutions in the lipopeptide surfactin using 2D NMR spectroscopy. Biochem. Biophys. Res. Commun. 1991, 177, 998-1005.

24. Heerklotz, H.; Seelig, J. Leakage and lysis of lipid membranes induced by the lipopeptide surfactin. Eur Biophys J. 2006, 35, 305-314.

25. Meta, M.; Yang, S.H.; Bergo, M.O.; Fong, L.G.; Young, S.G. Protein farnesyltransferase inhibitors and progeria. Trends Mol. Med. 2006, 12, 480-487.

26. Cao, X.H., Wang, A.H., Wang, C.L., Mao, D.Z., Lu, M.F., Cui, Y.Q., \& Jiao, R.Z. (2010). Surfactin induces apoptosis in human breast cancer MCF-7 cells through a ROS/JNK-mediated mitochondrial/caspase pathway. Chem Biol Interact., 183, 357-362.

27. Wakamatsu, Y.; Zhao, X.; Jin, C.; Day, N.; Shibahara, M.; Nomura, N. Manno- 
sylerythritol lipid induces characteristics of neuronal differentiation in PC12 cells through an ERK-related signal cascade. Eur. J. Biochem. 2001, 268, 374-83.

28. Zhao, X.; Wakamatsu, Y.; Shibahara, M.; Nakahara, N.; Geltinger, C.; Nakahara, T. Mannosylerythritol lipid is a protent inducer of apoptosis and differentiation in PC12 cells through and ERK-related signal cascade. Eur J Biochem. 2001, 268, 374-83.

29. Sudo, T.; Zhao, X.; Wakamatsu, Y.; Shibahara, M.; Nakahara, T. Induction of the differentiation of human HL-60 promyelocytic leukemia cell line by succinoyl trehalose lipids. Cytotechnology 2000, 33, 259-64.

30. Wang, C.L.; Ng, T.B.; Yuan, F.; Liu, Z.K.; Liu, F. Induction of apoptosis in human leukemia K562 cells by cyclic lipopeptide from Bacillus subtilis natto T-2. Peptides 2007, 28, 1344-1350.

31. Cao, X.H.; Wang, A.H.; Jiao, R.Z.; Wang, C.L.; Mao, D.Z.; Yan, L.; Zeng, B. Surfactin induces apoptosis and G2/M arrest in human breast cancer MCF-7 cells through cell cycle factor regulation. Cell Biochem. Biophys. 2009, 55, 163-171.

32. Ohno, A.; Ano, T.; Shoda, M. Production of a lipopeptide antibiotic surfactin with recombinant Bacillus subtilis. Biotechnol. Lett. 1992, 14, 1165-1168.

33. Zhu, Z.; Zhang, F.; Wei, Z.; Ran, W.; Shen, Q. The usage of rice straw as a major substrate for the production of surfactin by Bacillus amyloliquefaciens XZ-173 in solidstate fermentation. J. Environ. Manage. 2013, 127, 96-102. 
34. Slvinski, C.T.; Mallmann, E.; de Araújo, J.M.; Mitchell, D.A.; Krieger, N. Production of surfactin by Bacillus pumilus UFPEDA 448 in solid-state fermentation using medium based on okara with sugarcane bagasse as a bulking agent. Proc. Biochem. 2012, 47, 2012, 1848-1855. 


\section{Figure captions}

Figure 1. TLC (A) and HPLC (B) profile of the surfactin produced by Bacillus pumilus HY1.

(A) Developing solvent: chloroform:methanol:water=65:25:4 (v/v/v) drops, Visualization:

$10 \% \mathrm{H}_{2} \mathrm{SO}_{4}$ in water and (B) Eluent solvent: acetonitrile/water $=1: 1(\mathrm{v} / \mathrm{v})$, flow rate: 2.5 $\mathrm{mL} / \mathrm{min}$. Absorbance: $214 \mathrm{~nm}$.

Figure 2. MALDI-TOF mass spectrum of the surfactin produced by Bacillus pumilus HY1.

The lipopeptide appeared as a complex mixture of several isoforms. Sodium (Na), potassium $(\mathrm{K})$, and calcium $(\mathrm{Ca})$ ions were detected.

Figure 3. ESI tandem mass spectrometry fragmentation of surfactin isomers. Peptide sequences of five surfactin isomers were identified based on the MS/MS spectrum of the precursor ion $\mathrm{m} / \mathrm{z}$ 994.7081, 1,008.7334, 1,022.7391, 1,036.7528, and 1,050.7487.

Figure 4. Changes in the visible cell number and surfactin concentration during fermentation of chenonggujang by Bacillus pumilus HY1.

Figure 5. Cell population of human breast (MCF-7) and intestinal (Caco-2) cancer cell lines 
according to surfactin concentration. (A) Surfactin extraction of cheonggukjang-treated MCF-7 cells and (B) surfactin extraction of cheonggukjang-treated Caco-2 cells.

Figure 6. Cytotoxicity test of human breast (MCF-7) and intestinal (Caco-2) cancer cell lines by adding surfactin extraction of cheonggukjang. (A1) MCF-7 cells (control), (A2) surfactin extraction of cheonggukjang-treated MCF-7 cells (100 $\mu \mathrm{g} / \mu \mathrm{l})$, (B1) Caco-2 cells (control) and (B2) surfactin-treated Caco-2 cells $(100 \mu \mathrm{g} / \mu 1)$.

\section{Table caption}

Table 1. ICP mass spectrum of surfactin isomers from surfactin produced by Bacillus pumilus HY1. 


\section{Figure 1}

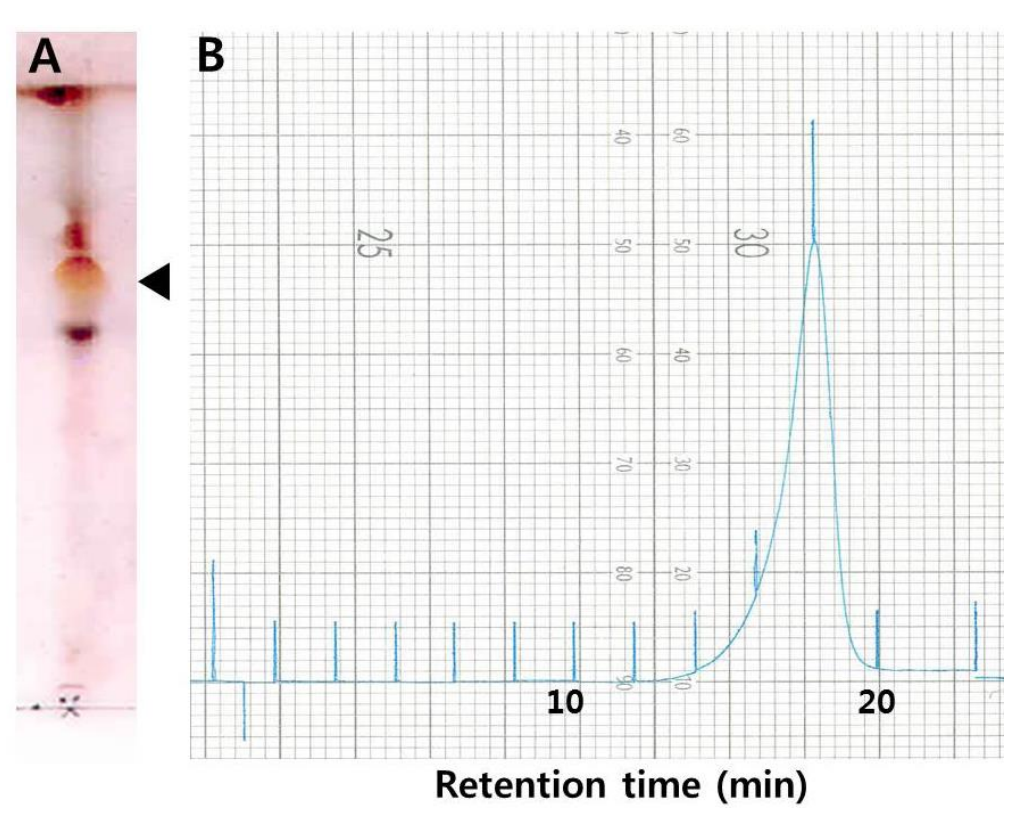




\section{Figure 2}

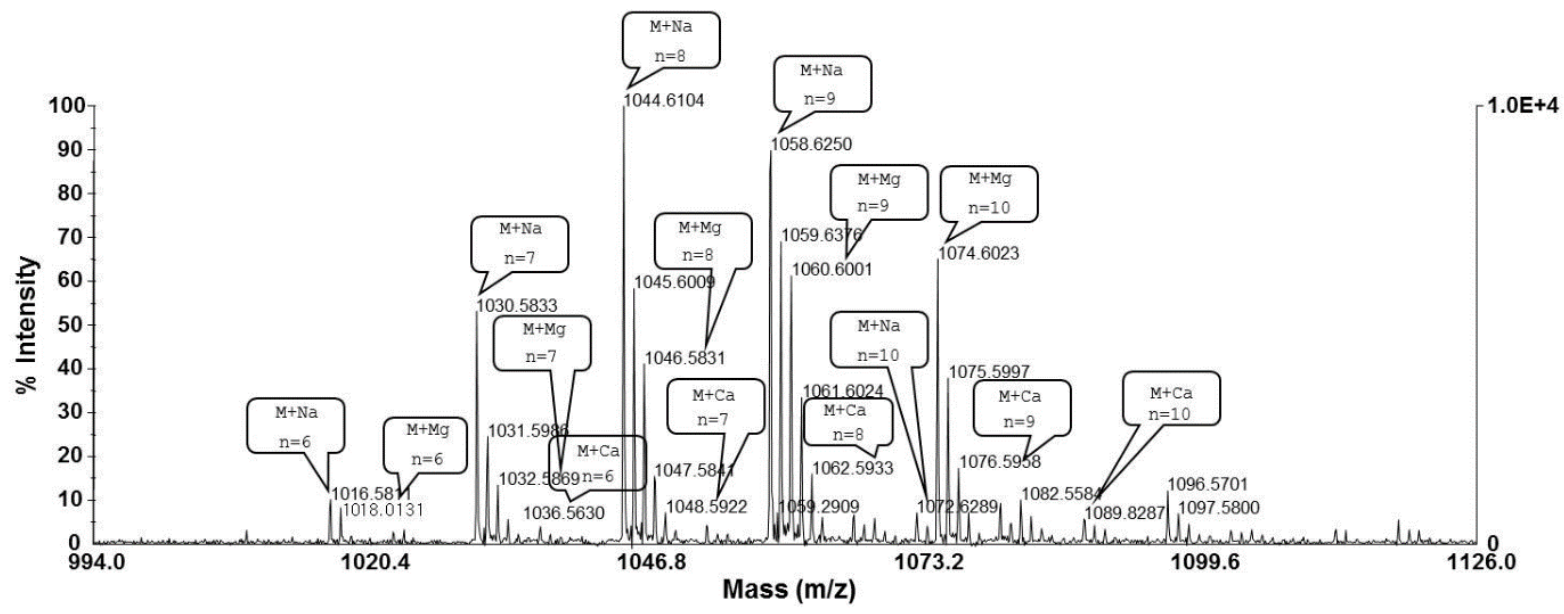




\section{Figure 3}
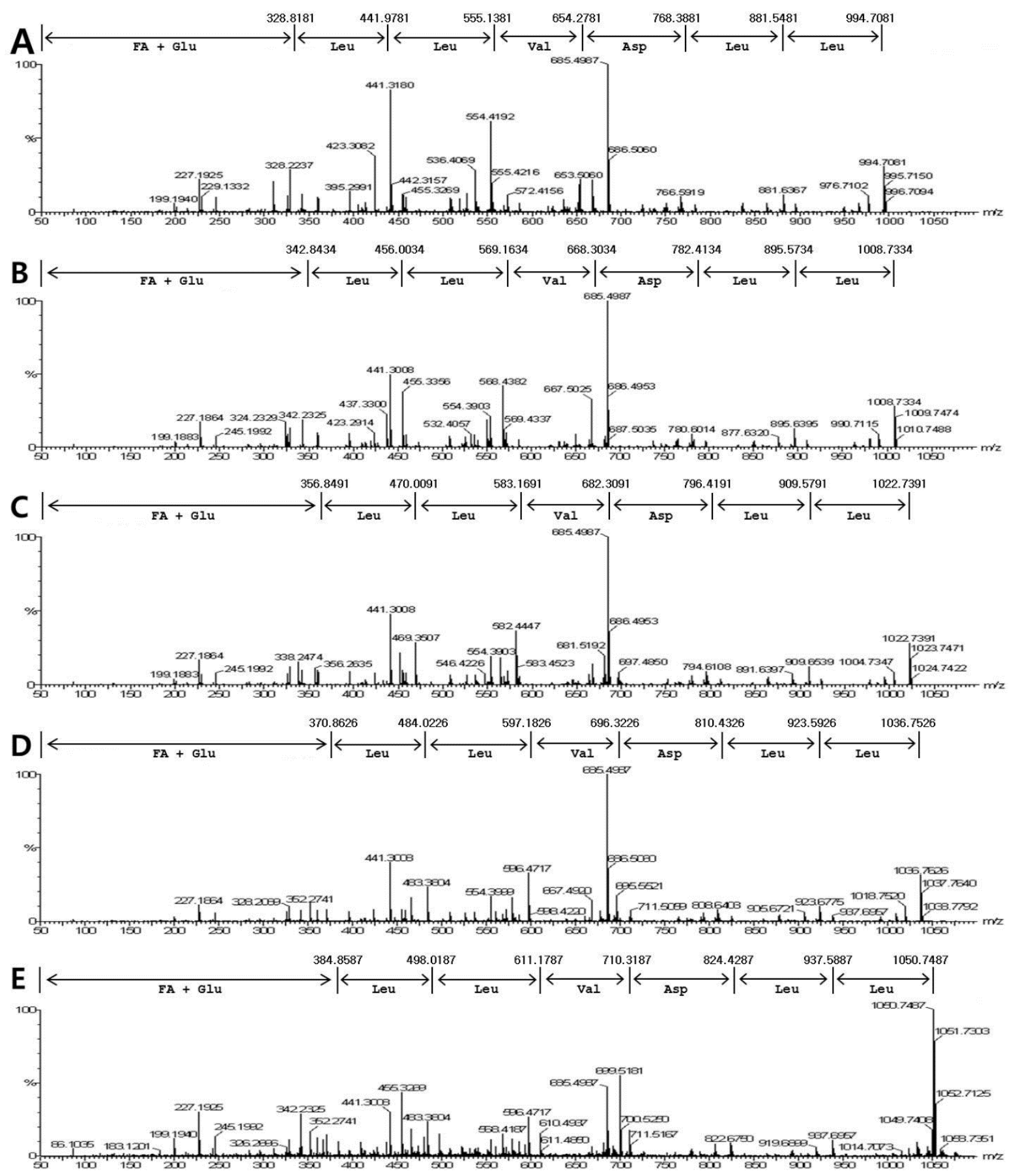


\section{Figure 4}

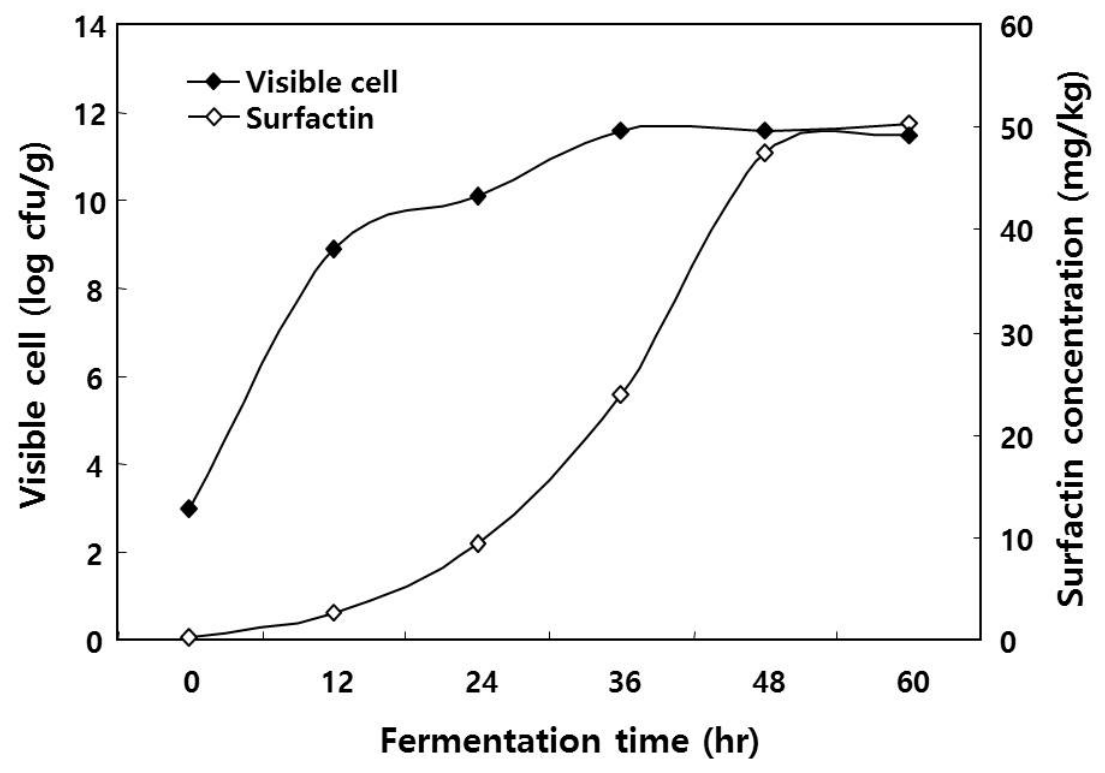




\section{Figure 5}
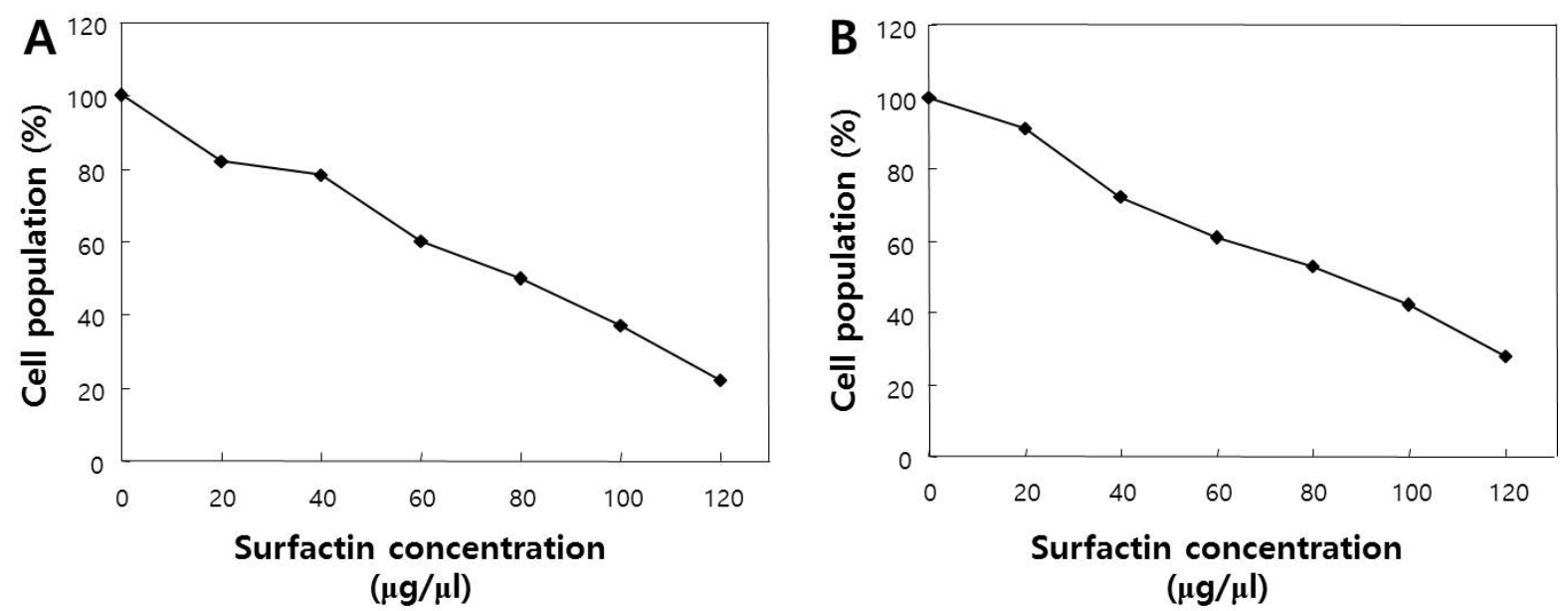


\section{Figure 6}

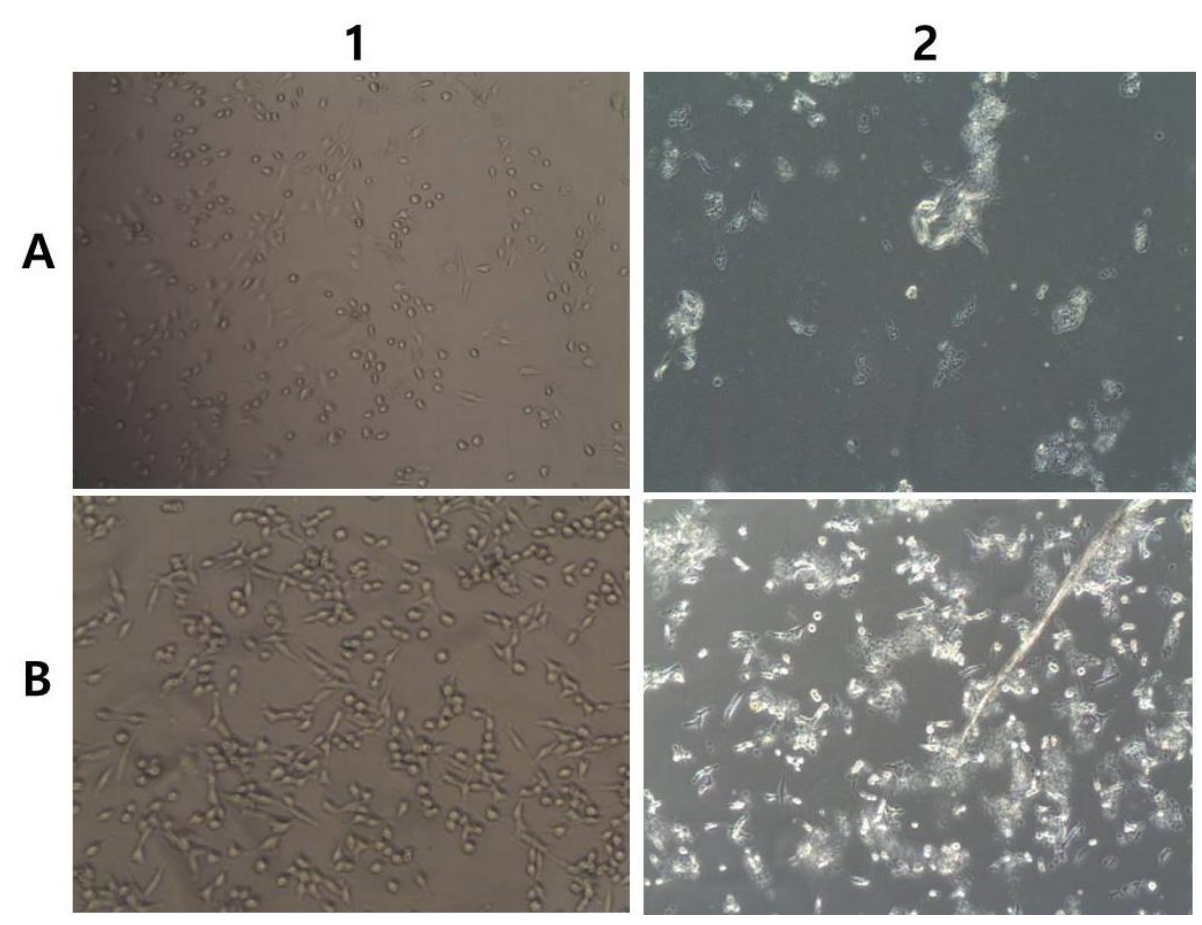




\section{Table 1}

ICP mass spectrum of surfactin isomers from surfactin produced by Bacillus pumilus HY1.

\begin{tabular}{|c|c|c|c|}
\hline Elements & Concentration (ppb) & Elements & Concentration (ppb) \\
\hline $\mathrm{Al}$ & 0.0 & $\mathrm{P}$ & 1121.5 \\
\hline B & 133.6 & $\mathrm{Rb}$ & 0.0 \\
\hline $\mathrm{Br}$ & 0.0 & $\operatorname{Re}$ & 0.0 \\
\hline $\mathrm{Ca}$ & 1509.6 & $\mathrm{Ru}$ & 94.4 \\
\hline $\mathrm{Cd}$ & 0.0 & S & 348218.9 \\
\hline $\mathrm{Cr}$ & 122736.5 & Sc & 74514.2 \\
\hline Cs & 0.0 & $\mathrm{Se}$ & 1112974.3 \\
\hline $\mathrm{Cu}$ & 0.0 & $\mathrm{Sn}$ & 115.1 \\
\hline $\mathrm{Ge}$ & 194.9 & $\mathrm{Sr}$ & 0.0 \\
\hline $\mathrm{Hg}$ & 26.9 & $\mathrm{Ta}$ & 2.9 \\
\hline I & 1375.0 & $\mathrm{Ti}$ & 580.2 \\
\hline K & 89.4 & $\mathrm{U}$ & 0.0 \\
\hline $\mathrm{Li}$ & 122.4 & V & 0.0 \\
\hline $\mathrm{Mg}$ & 5634.3 & $\mathrm{~W}$ & 44.7 \\
\hline $\mathrm{Mn}$ & 0.0 & $\mathrm{Y}$ & 538.1 \\
\hline Mo & 0.0 & $\mathrm{Yb}$ & 0.0 \\
\hline $\mathrm{Na}$ & 6446.2 & $\mathrm{Zn}$ & 2351.4 \\
\hline Os & 0.0 & & \\
\hline
\end{tabular}

